\title{
Optimization of the operation of a fiber optic interference-based sensor for perimeter protection
}

\author{
M. Życzkowski \& P. Markowski \\ Institute of Optoelectronics, Military University of Technology, Poland
}

\begin{abstract}
The main purpose of the paper is to present the impact of the polarization state of light in fiber optic interference sensors on their stability and efficiency. The main application of interference-based sensors for security is the protection of wide area objects, especially perimeter protection. This type of sensor offers high accuracy of locating disturbances and a high certainty of detection. Their advantage is resistance to external electromagnetic interference, such as storms, or to influence other devices located in the detection zone. The sensitive part of the sensor does not introduce electromagnetic disturbances to the environment. An interference-based fiber sensor requires complex methods of signal detection in order to achieve the maximum efficiency. The paper describes research on the impact of polarization state of light in fiber optic interference-based sensors on stability, accuracy and efficiency in order to determine the alarm state and the disorder location. The paper presents a method to stabilize the state of light polarization in order to maintain the detection state of the maximum contrast. We determined the state of light polarization with the highest detection efficiency and stability of tested sensors. We present an interference-based sensor with a stabilized level of detection at the point of the maximum sensitivity.

Keywords: infrastructure protection, fiber optic sensor, interference-based sensors.
\end{abstract}

\section{Introduction}

At the time of the ubiquitous threat of terrorist attacks around the world, it is essential to ensure the security of critical infrastructure facilities. Perimeter fencing is the primary barrier in the way of an intruder while attempting 
intrusion on a protected area. To ensure the quickest response of security services, fence monitoring systems are applied which are able to detect and locate the place of intrusion. Due to the fact that elements of this type of detection systems are the first line of protection, it is necessary to make information provided by the system the most certain alike in terms of sensitivity, detection and the minimum number of false positives, as well as in terms of resistance to environmental factors. By analysing the above requirements it becomes clear that the interference-based sensors are the best solution. They offer a high sensitivity, the ability to locate, as well as they are resistant to external atmospheric conditions. Unfortunately, this type of sensors require complex detection and control systems to ensure that they are up to their optimum use. By controlling and adjusting operating parameters of interferencebased sensors, it is possible to adapt the system to work in variable environmental conditions with maximum sensitivity to intrusion occurrence.

The paper presents the method for automatic correction of the input state of light polarization of the double Mach-Zehnder interferometer. Correction of polarization state at the input light aims to generate and maintain interference contrast with a value close to one. The presented system is dedicated to applications in the field of security systems [1] such as perimeter security of critical infrastructure or monitoring integrity of telecommunication lines [2]. The system can successfully be used in other applications requiring detection of mechanical [3] disorders along many kilometers zone with the possibility of determining the place of disorder [4].

The presented system was tested in field conditions. The aim of the measurements was to determine the possibility of detection disorders along the fiber optic cable and stability of the system.

\section{Influence of light polarization on the interference pattern}

Interference pattern can be generated only by a coherent light [5]. The main parameter which determines the obtained interference pattern is phase difference of two mutually interfering light waves. The phase difference is a result of the difference in optical length of the interferometer arms. If the resultant phase difference is equal to zero, a constructive interference occurs resulting in the maximum possible light amplification. If the resultant phase difference of two interfering waves is equal to $\pi$, a destructive interference occurs and a complete extinction of the light could take place. In other cases, the observed intensity at the output can have a value between the intensity of the maximum gain and the intensity of total extinction.

In the case of light interference, polarization state is also crucial [6]. For orthogonally polarized waves interference does not occur. For the case when states of polarization of two interfering waves are non-orthogonal, it is possible to observe interference fringes. The maximum contrast of the interference can be achieved only for waves with the same state of polarization.

A qualitative parameter describing the interference pattern is the interference contrast [7]. Contrast is defined as the ratio of a difference between maximum 
and minimum values of light intensity and a sum of maximum and minimum values of light intensity. Mathematical relation that defines the interference contrast describes formula (1)

$$
K=\frac{I_{\max }-I_{\min }}{I_{\max }+I_{\min }}
$$

where: $\mathrm{K}$ - interference contrast, $\mathrm{I}_{\max }$ - maximum value of light intensity, $I_{\min }-$ minimum value of light intensity.

In the fiber-optic interferometer systems constructed on the basis of a standard cylindrical fiber, state of polarization changes with distance along the fiber. Wherein each fiber introduces various changes of polarization state which depend on local stress and torsion of fibers. Stress and torsion introduce significant birefringence of an optical fiber.

Through the proper selection of polarization state, interferometer system can affect the value of interference contrast. It is possible to distinguish two polarization states (mutually orthogonal) for which the contrast takes maximum values. For these states shifts of polarization in both arms of the interferometer are the same, thus, interfering waves are polarized equally at the output of interferometer. Visualization of the impact of input polarization state on the interference contrast is presented in figure 1.
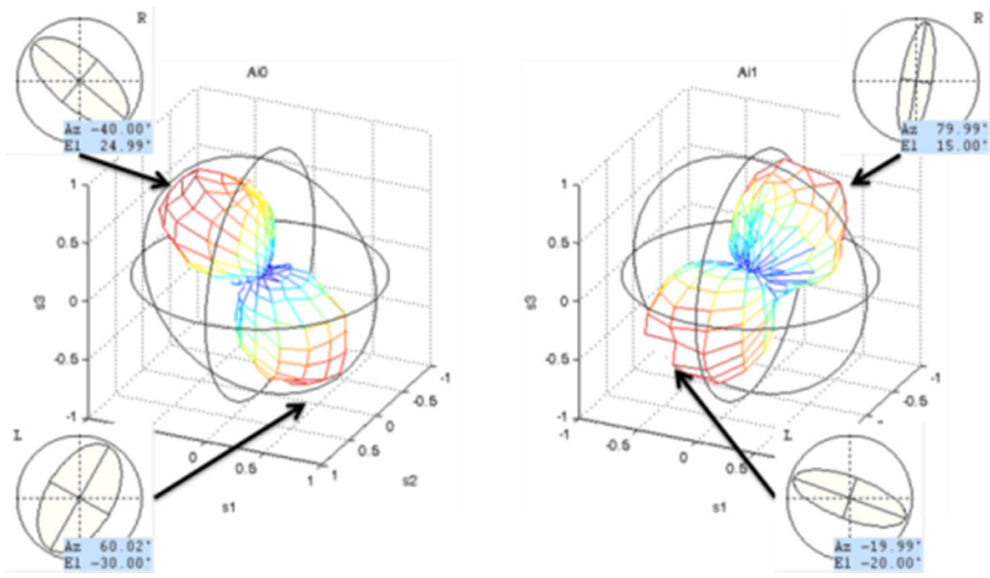

Figure 1: Influence of input state of polarization on the output interference contrast.

A change of polarization state at the input of interferometric system (for which the maximum contrast is achieved) may be caused by the change of propagation conditions. Polarization states for which the maximum contrast is achieved remain orthogonal to each other. The shift of state of polarization (for which the maximum contrast is achieved) is greater as greater is the change in propagation conditions. 


\section{Construction and principle of operation of the double Mach-Zehnder interferometer}

The presented optic interferometer system is built on the basis of the MachZehnder interferometer. An optical supply is applied at both ends of the system in the opposite direction of light propagation: clockwise $\mathrm{CW}$ and counter clockwise CCW. A schematic diagram of the system is shown in figure 2.

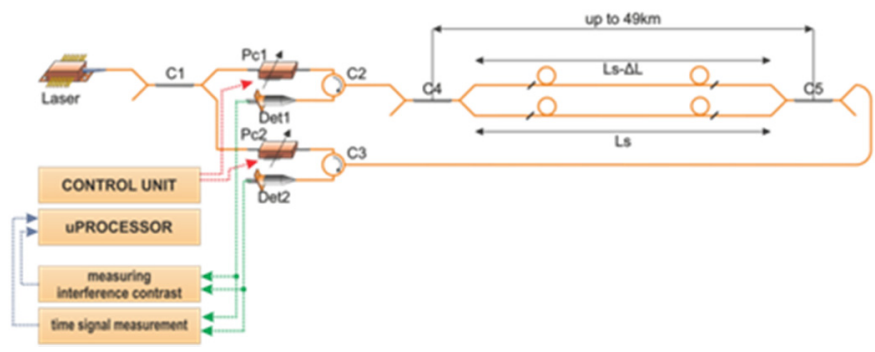

Figure 2: Schematic diagram of the double Mach-Zehnder interferometer.

The presented system can be divided into two parts: control - transmitting receiving optoelectronic systems and passive fiber optic part. The elements of control - transmitting - receiving optoelectronic systems are: coherent light source with modulated wavelength (fiber laser THE ROCK NP Photonics controlled by a generator Tektronix AFG3021.) $2 \times 2$ 3dB coupler, two polarization controllers (PCD-M02-B controlled with the NI USB 6009 card), two detectors (Thorlabs DET01CFC NI PCI-6115 with input/output block NI BNC-2110) and PC unit with LabView software. The built laboratory system is shown in figure 3.

In the presented double interferometer system both Mach-Zehnder interferometers have the same properties in terms of sensitive parts of the system. Arms of both interferometers have exactly the same length, thus the imbalance between the arms of two interferometers is the same. Sensitive parts introduce the same stress and, as a result, both systems provide the same interference pattern.

Direction of light propagation is opposite in both interferometers. Length of optical supply path and length of receiving path are also different.

Both optical systems are supplied by the same source which generates the light with a constant wavelength modulation. The optical radiation is separated by a $2 \times 23 \mathrm{~dB}$ coupler and transmitted to polarization controllers. The independent state of polarization for each direction of propagation is set to achieve the maximum contrast of interference pattern at the output. Then, the radiation is transmitted to the Mach-Zehnder interferometer - for the CW direction from polarization controller by the circulator $\mathrm{C} 2$ directly to the arms of interferometer, and for the $\mathrm{CCW}$ direction through the circulator $\mathrm{C} 3$, then through a fiber along the interferometer's arms. As a result, the interferometric pattern is transmitted to the detector - for the $\mathrm{CW}$ direction pattern is transmitted 


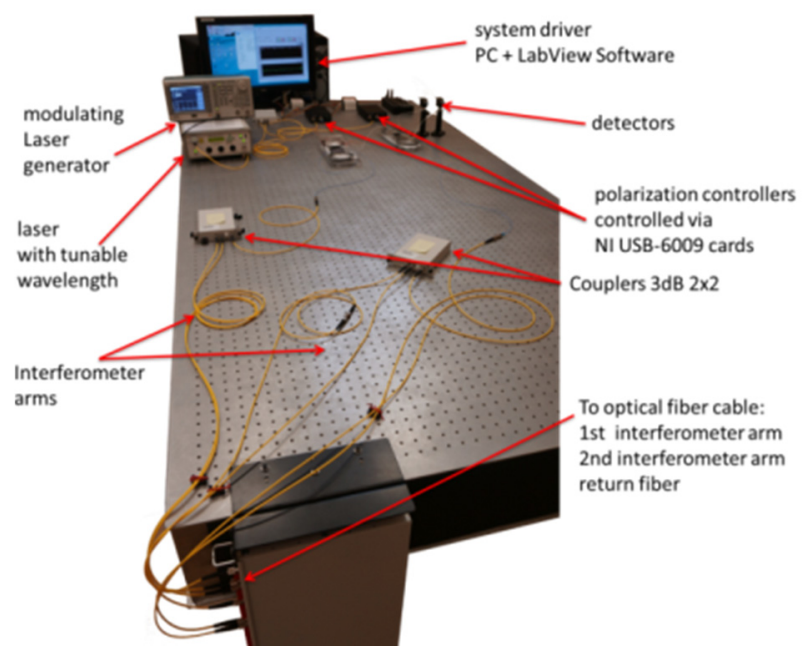

Figure 3: Operated laboratory system of double Mach-Zehnder interferometer.

through a fiber along the interferometer's arms, by the circulator $\mathrm{C} 2$ to detector, and for the $\mathrm{CCW}$ direction it is transmitted by the circulator $\mathrm{C} 3$ directly to the detector.

In order to perform continuous measurement of interference contrast, a modulation of the operation point is introduced to cover the entire transfer characteristics of the system. Modulation parameters should be selected according to an aligned optical line. For a system with a non-zero optical path difference relation for desired depth of wavelength modulation describes formula (2).

$$
\Delta \mathrm{V}=\frac{\mathrm{c} \cdot \Delta \varphi}{2 \pi \cdot \mathrm{n}_{\mathrm{co}} \Delta \mathrm{L}}
$$

where: $\Delta \mathrm{V}$-depth of wave modulation, $\mathrm{c}-$ speed of light, $\Delta \varphi-$ position of the middle of operating point in transfer function graph, $\mathrm{n}_{\mathrm{co}}-$ refractive index of fiber core, $\Delta \mathrm{L}$ - optical path difference of interferometer's arms.

For such defined modulation depth a function graph describing the relationship between the required retuning of the laser and the optical path difference can be plotted. Relationship between the values of optical path imbalance of the interferometer's arm and the required tuning range wavelengths of the supply system is shown in figure 4 .

Selection of modulation parameters affects the stability and reliability of the measurement interference contrast pattern. According to equation (2), the modulation depth depends on imbalance of the interferometer's arms' length. An insufficient modulation depth can cause the transfer function graph to be not fully covered. It may be impossible to achieve the maximum possible contrast and the interference pattern may be unstable and highly variable. For a too high 
depth of modulation the operating point may cover the transfer function graph several times for one full period of wavelength change. In this case, the interference contrast reaches the maximum value for a given state of polarization, but the contrast of the interference may fluctuate.

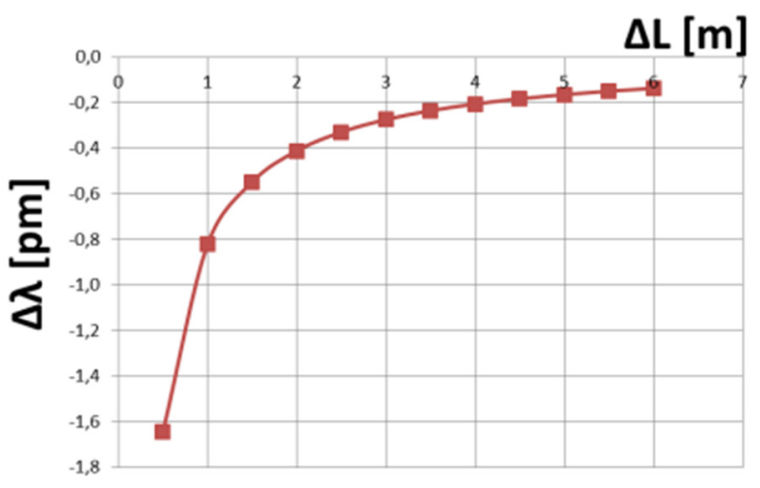

Figure 4: Depth of modulation of the input light as a function of unbalance of optical path length.

It is possible to distinguish a certain value of modulation depth for the maximum contrast and minimal fluctuations. This is exactly the value of modulation depth corresponding to the length of the interferometer's arms' imbalance as a result of the equation (2). A graph of medium contrast, standard deviation and difference of the measured maximum and minimum contrast with 1000 series of measurements for each value of voltage controlling the modulation depth for both directions at the same time is shown in figure 5.
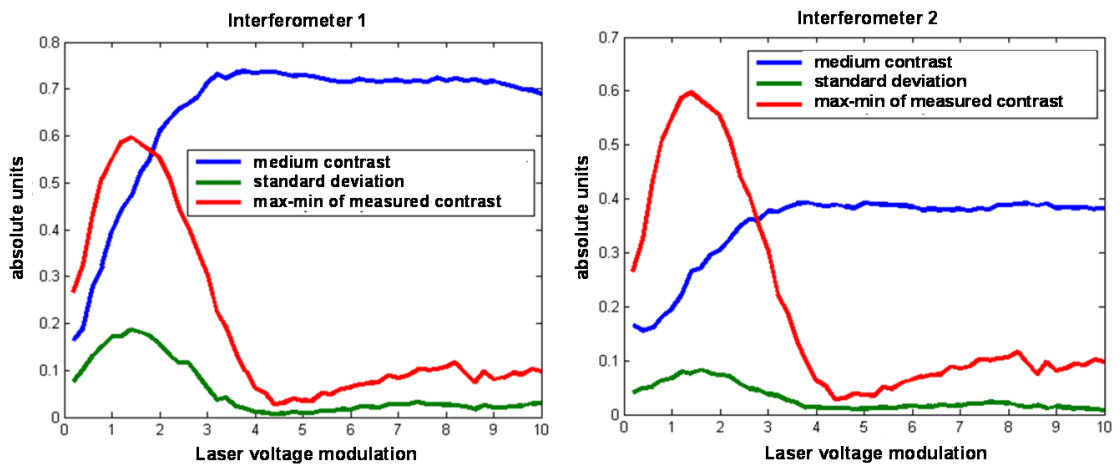

Figure 5: Medium contrast, standard deviation and difference of the measured maximum and minimum contrast with 1000 series of measurements for each value of voltage controlling the modulation depth for both directions. 
For both systems it is possible to select the value of modulation depth to maximize contrast of the interference and to minimize the fluctuations. Because of that for both directions $\mathrm{CW}$ and $\mathrm{CCW}$ the value of imbalance length is identical and the modulation depth is the same for both directions. However, because two interferometers are supplied through different optical paths, an independent control of the input polarization states for both directions of propagation is required.

\section{Implementation of the polarization correction state}

In the described double Mach-Zehnder interferometer an alarm signal is generated based on the interference contrast. Due to the character of the system's application, the system is under continuous low frequency environmental disturbances causing small changes of values in the interference contrast. In order to improve detection parameters the maximum interference contrast is achieved by setting up the input state of polarization. All external disturbances can cause a decrease of contrast by introducing tension to the measurement channel. These changes can cause changes of polarization and phase at the end of the interferometer's arms. Changes in the range between 1 and 0.9 are categorized as noise, and the system does not respond. For the contrast values between 0.9 and 0.75 , the system is set to work with environmental impact and polarization state of the input light is set to achieve the maximum contrast, thus an alarm signal is not generated. Contrast values below 0.75 generate an alarm signal and the system recognizes this type of disorder as a physical interference with the optical path. Illustration of contrast changes and the required search area for optimal value of polarization state is shown in figure 6 .

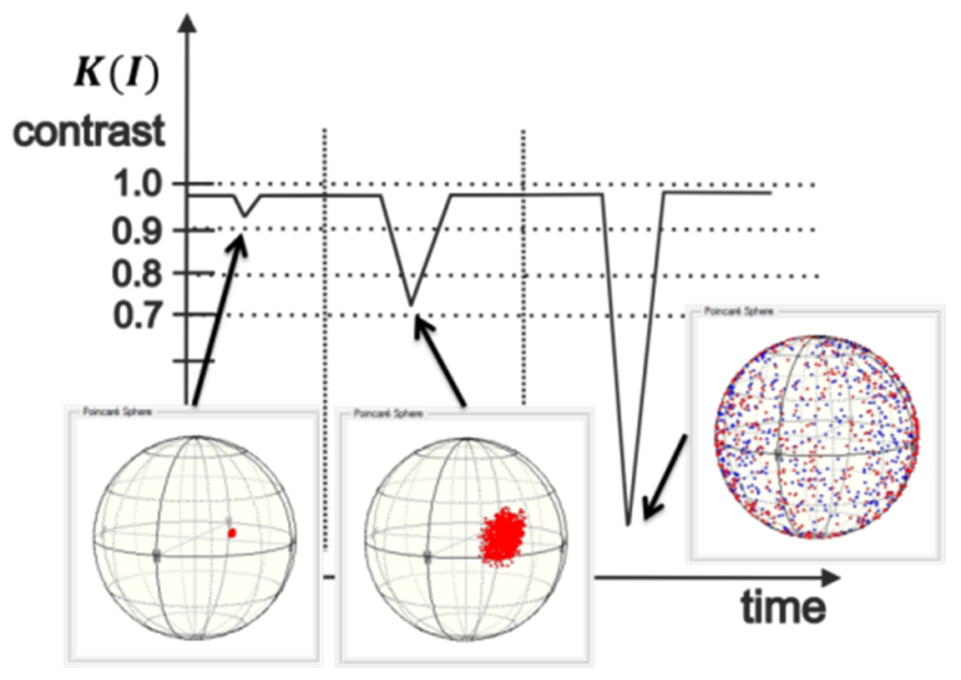

Figure 6: Contrast changes and the required search area of Poincare sphere for optimal value of polarization state. 
The adjustment algorithm of the input polarization state in the described system was implemented in a LabView 2013 environment. At the time of decrease of contrast the system corrects the input state of polarization. This is accomplished by setting random polarization states of the appropriate area in the Poincare sphere. Therefore, the basis of polarization state and the corresponding interference contrast are generated. After a predetermined number of scans the state of polarization with the maximum contrast value is set. The algorithm presenting the procedure of creating the basis of polarization states is shown in figure 7.

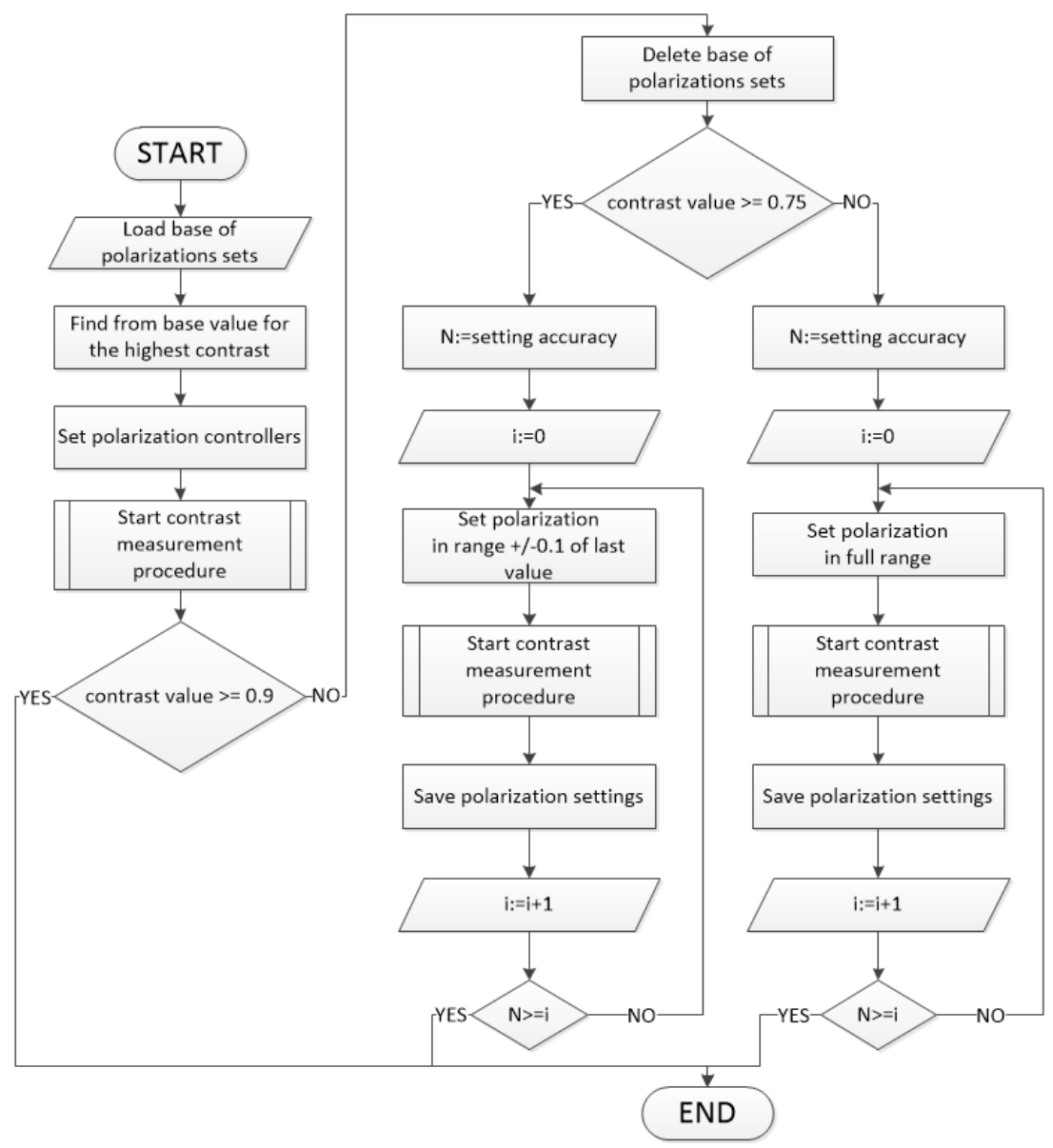

Figure 7: Poincare sphere searching algorithm to determine the base polarization states and the corresponding contrasts.

The system has been tested on the prepared test training ground built with standard telecommunication cables. Images presenting test environment and the running system are shown in figure 8. 


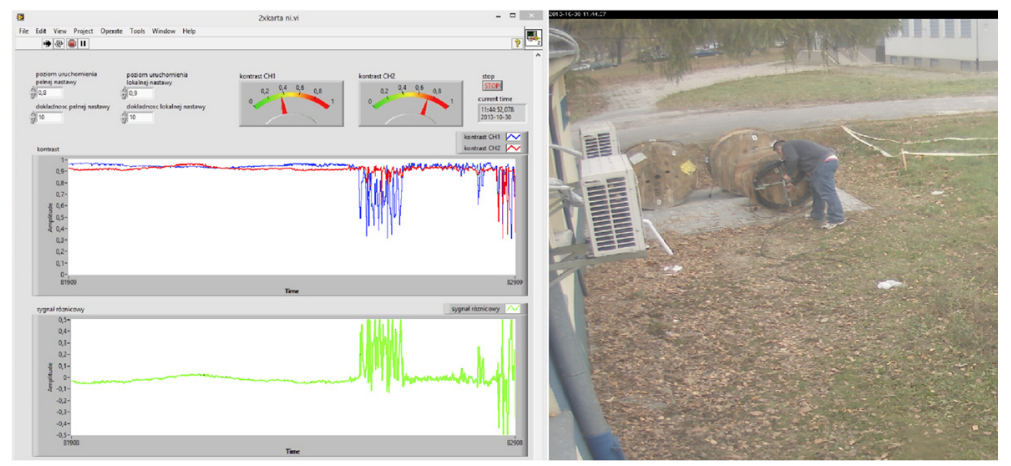

Figure 8: Fiber optic sensor system in a double configuration of the MachZehnder interferometer during field tests.

\section{Conclusions}

The presented system is stabilized to environmental vibrations, self-adjusting its parameters to temporary changes introduced by the environment. Therefore, the system generates an alarm signal as a response to the dynamic changes caused by a sudden appearance of external disturbances, such as physical contact with an optical fiber sensing cable. The tests demonstrate time stability of the system's operation with a proper detection of the external mechanical disturbances. In order to generate a signal suitable for measuring the contrast of the interference pattern, the system generates interference fringes with a frequency of $5 \mathrm{kHz}$. This allows to generate an alarm signal with a high probability as the frequency of signals generated by physical disturbances are in the range of $0-700 \mathrm{~Hz}$. Sampling rate of detection of electrical signals from the detectors was $100 \mathrm{kS} / \mathrm{s}$.

\section{Acknowledgement}

The project is co-financed by The National Centre for Research and Development within the project realized for national security and defence "Fiberoptic link integrity monitoring system for protection against unauthorised access to classified information" (System monitorowania integralności łącza światłowodowego w celu ochrony przed nieautoryzowanym dostępem do informacji niejawnych) Contract no. DOBR/0070/R/ID1/202/03.

\section{References}

[1] M. Szustakowski, W. Ciurapinski, M. Życzkowski, J. Wrobel, R. Dulski, P. Markowski, Security-orientated Plastic Optical Fiber sensor in Modalmetric Configuration, Procedia Engineering, 47, pp. 916-923, 2012

[2] M. Życzkowski, M. Szustakowski, W. Ciurapinski, P. Markowski, M. Karol, M. Kowalski, Optical Fiber Sensors as the Primary Element in the 
Protection of Critical Infrastructure especially in Optoelectronic Transmission Lines, Safety and Security Engineering V, pp. 273-283, 2013

[3] J. Dakin; B Culshaw, Optical fiber sensors: Systems and applications, Artech House, Boston 1989

[4] M. Życzkowski, M. Kondrat, W. Ciurapinski, Initial research of dual wavelength fiber optic perimeter sensor, Journal de physique. IV, JP 129, pp. 189-191, 2005

[5] H. F. Schouten, G. Gbur, T. D. Visser, E. Wolf, Phase singularities of the coherence functions in Young's interference pattern, Optics Letters, 28(12), pp. 968-970, 2003

[6] Nori Shibata, Akimichi Nakazono, Yoshinori Inoue, Interference between two orthogonally polarized modes traversing a highly birefringent air-silica microstructure fiber, Journal of Lightwave Technology, 23(3), pp. 12441252, 2005

[7] Wu Yuan, Bian Pang, Jia Bo and Xiao Qian, Fiber-optic sensor without polarization-induced signal fading, Microwave and Optical Technology Letters, 56(6), pp. 1307-1313, 2014 